\title{
OA05.02. Relationships among well-being and wellness behaviors over time in residents in eight family medicine residencies
}

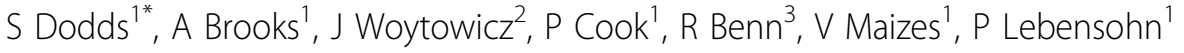 \\ From International Research Congress on Integrative Medicine and Health 2012 \\ Portland, Oregon, USA. 15-18 May 2012
}

\section{Purpose}

To present findings on relationships among dimensions of well-being and wellness behaviors in family medicine residents participating in the Integrative Medicine in Residency (IMR) program through the last two years of training.

\section{Methods}

Residents in the 2011 graduating class of the IMR $(n=56)$ were assessed at the beginning of PGY2 and PGY3 and at graduation. Measures were self-administered online and included established measures of wellbeing: perceived stress, burnout (emotional exhaustion, depersonalization), depression, and satisfaction with life. Wellness behaviors assessed included restful sleep, nutrition, physical activity, mind-body activities, being in nurturing relationships, being outdoors in nature, and alcohol use. Stepwise regression analysis examined relationships between wellness behaviors and each wellbeing measure at each timepoint.

\section{Results}

In both PGY2s ( $\mathrm{n}=52)$ and PGY3s $(\mathrm{n}=38)$, restful sleep was associated with less perceived stress $(p=0.003$; $\mathrm{p}=0.01)$, greater life satisfaction $(\mathrm{p}=0.007 ; \mathrm{p}=0.007)$, less depression $(\mathrm{p}=0.002 ; \mathrm{p}=0.041)$, and less emotional exhaustion ( $\mathrm{p}=0.001 ; \mathrm{p} \leq 0.001)$. In PGY2s, more time in nurturing relationships was associated with greater life satisfaction $(\mathrm{p}=0.039)$. In PGY3s, more frequent exercise was associated with less depression $(p=0.003)$ and greater life satisfaction $(\mathrm{p}=0.014)$. By graduation $(\mathrm{n}=42)$, sleep was associated with less emotional exhaustion $(\mathrm{p}=0.006)$. Spending more time outdoors in nature was associated with lower perceived stress $(\mathrm{p}=0.002)$, less depression $(\mathrm{p}=0.026)$, and lower depersonalization $(\mathrm{p}=0.003)$. Nurturing relationships were associated with greater life satisfaction $(\mathrm{p} \leq 0.001)$. More alcohol use was associated with less perceived stress $(\mathrm{p}=0.001)$. Using a variety of mind-body wellness behaviors was associated with greater depression in PGY2s $(\mathrm{p}=0.015)$, and with emotional exhaustion at graduation $(\mathrm{p}=0.045)$.

\section{Conclusion}

Sleep, nurturing relationships, exercise, and time outdoors in nature were most frequently associated with well-being among Family Medicine residents. This study is the first to describe these relationships for residents who participated in the IMR, and points to the importance of addressing well-being and wellness behaviors during the formative time of graduate medical education.

\section{Author details \\ 'University of Arizona Center for Integrative Medicine, Tucson, USA. ${ }^{2}$ Maine- Dartmouth, Augusta, USA. ${ }^{3}$ University of Michigan Health Center, Ann Arbor,} USA.

Published: 12 June 2012

doi:10.1186/1472-6882-12-S1-018

Cite this article as: Dodds et al:: OA05.02. Relationships among wellbeing and wellness behaviors over time in residents in eight family medicine residencies. BMC Complementary and Alternative Medicine 2012 12(Suppl 1):018.

'University of Arizona Center for Integrative Medicine, Tucson, USA

Full list of author information is available at the end of the article

(C) 2012 Dodds et al; licensee BioMed Central Ltd. This is an Open Access article distributed under the terms of the Creative Commons Attribution License (http://creativecommons.org/licenses/by/2.0), which permits unrestricted use, distribution, and reproduction in any medium, provided the original work is properly cited. 